\title{
Vingt ans de réformes foncières en Europe centrale et orientale. Bilan et perspectives
}

Twenty years of land reforms in Central and Eastern Europe: state of play and outlook

\section{Céline Bignebat et Laure Latruffe}

\section{OpenEdition}

Journals

Édition électronique

URL : http://journals.openedition.org/economierurale/3225

DOI : 10.4000/economierurale.3225

ISSN : 2105-2581

Éditeur

Société Française d'Économie Rurale (SFER)

Édition imprimée

Date de publication : 21 octobre 2011

Pagination : 25-38

ISSN : 0013-0559

Référence électronique

Céline Bignebat et Laure Latruffe, «Vingt ans de réformes foncières en Europe centrale et orientale. Bilan et perspectives », Économie rurale [En ligne], 325-326 | septembre-décembre 2011, mis en ligne le 17 octobre 2013, consulté le 20 avril 2019. URL : http://journals.openedition.org/economierurale/3225 ; DOI : 10.4000/economierurale.3225 


\title{
Vingt ans de réformes foncières en Europe centrale et orientale Bilan et perspectives
}

\author{
Céline BIGNEBAT •INRA, UMR 1100 MOISA, Marchés, Organisations, Institutions et Stratégies \\ d'Acteurs, Montpellier
}

Laure LATRUFFE • INRA, UMR1302 SMART, Structures et Marchés agricoles, Ressources et Territoires, Rennes

a transition économique dans les Pays
d'Europe centrale et orientale (PECO) impliquait une réallocation des ressources de façon à produire de manière plus efficace, et nécessitait la libéralisation des prix et des échanges, et la privatisation des moyens de production étatiques (voir Bignebat et al., dans ce numéro). Dans le secteur agricole, la privatisation passait par les réformes foncières, puisqu'en général la terre est le facteur de production principal. Les lois foncières ont été parmi les premières votées par les nouveaux gouvernements élus après la fin du régime centralisé, qui avaient bien conscience de la nécessité cruciale de la privatisation des terres pour maintenir la production alimentaire de leur pays. Différentes formes de privatisation ont été choisies par les pays d'Europe centrale et orientale (restitution ou/et redistribution), mais le bilan que l'on peut en tirer reste mitigé : d'une part, la fragmentation des terres due à la méthode de privatisation et les difficultés de restructuration parcellaire expliquent pour part la persistance de petites structures. D'autre part, les grandes structures, fermes d'État ou collectives, ont subsisté pendant la transition, instaurant parfois une bipolarisation des structures foncières.

Nous avançons l'hypothèse que, plus encore que dans les dynamiques observées pour les pays en développement où la privatisation de la terre est décrite comme une évolution endogène due à la pression foncière croissance, les conditions initiales de la transition des PECO jouent un rôle majeur dans l'évolution observée. Maurel (2006) conclut que le succès des réformes économiques dans ces pays dépend surtout de l'héritage historique et géographique. Dans le secteur agricole, la composante « firme » de la transition agricole, c'est-àdire la privatisation de fermes d'État et collectives dans leur ensemble (et non seulement la privatisation de la terre), peut créer un effet de blocage de la réallocation des facteurs de production qui a été documenté par la littérature, objet de la première partie de cet article. Un tel blocage est accentué par la dimension foncière des réformes menées. La partie suivante explique ces réformes foncières en insistant tout d'abord sur leur logique et leurs modalités. La troisième partie tire ensuite un bilan de ces réformes, au terme de deux décennies de transition. La conclusion développe quelques perspectives.

\section{Nécessité et modalités des réformes foncières}

\section{La nécessité économique}

De nombreux auteurs (Deininger et Feder, 2001 ou Binswanger et Rosenzweig, 1986) ont souligné l'intérêt économique des réformes foncières, et plus particulièrement l'intérêt économique de l'émergence de droits de propriété individuels, dans les pays en développement. Si cet intérêt prévaut également dans les pays qui ont connu jusqu'en 1989 un régime centralisé, les modalités des réformes ont en général été diffé- 
rentes, en raison d'une caractéristique propre à ces pays : la situation initiale.

\section{Droits de propriété et situation initiale}

Nous considérons ici comme la situation initiale celle qui prévalait avant le début de la période de transition. Mais, de fait, les situations initiales étaient variées. En effet, le processus de collectivisation des terres entamé après la Seconde Guerre mondiale dans les pays d'Europe centrale et orientale et dans les pays de l'Union soviétique ne s'est pas fait de manière homogène selon les pays. Civici et Jouve (2009) soulignent l'importance des résistances paysannes, des traditions historiques et des relations politiques avec le régime soviétique.

Ainsi, la Pologne et la Yougoslavie ont par exemple évité la collectivisation agraire de grande échelle et ont toujours maintenu un secteur agricole individuel important. Pour leur part, l'Union soviétique et l'Albanie ont nationalisé la terre. Enfin, la propriété de la terre avant la transition économique était dominée, pour le reste des pays d'Europe centrale et orientale, par un contrôle de facto de la propriété foncière par les fermes d'État et fermes collectives même si de jure, les droits de propriété étaient restés individuels, sauf dans quelques cas, comme celui de la Hongrie (Macours et Swinnen, 2002).

Dans la majeure partie des pays anciennement communistes, les grandes structures industrielles de production étaient privilégiées, sous deux formes possibles : des fermes d'État organisées selon les mêmes principes qu'une entreprise, où les employés recevaient un salaire et l'État détenait tous les droits de contrôle sur l'investissement et la production; des fermes collectives où la rémunération des travailleurs se faisait sur la production (Rozelle et Swinnen, 2004). Malgré des économies d'échelle importantes, de nombreuses études empiriques ont conclu à l'inefficacité de ces grandes structures (par exemple Brada et King, 1993). En effet, la séparation entre propriété et management signifiait de trop faibles incitations à l'effort perçues par les travailleurs, dont le revenu n'était que peu lié à la performance de l'exploitation ; de plus, les difficultés de supervision du travail dans ces structures de grandes tailles créaient de forts coûts de transaction (Pollak, 1985 ; Schmitt, 1991).

Enfin, de nombreuses fermes collectives et d'État produisaient à perte, n'ayant aucune menace crédible de banqueroute ou de pénalités au non-remboursement des prêts accordés par la banque centrale : l'État accordait en effet de généreuses subventions publiques et autorisait le recul des échéances de remboursement des dettes (la contrainte de budget molle définie par Kornai, 1980). Le secteur agricole n'était pas le seul à être concerné par cette situation. Le début de la transition économique est marqué par la mise en œuvre de réformes visant à supprimer les distorsions de prix induites par le système centralisé et à rétablir des incitations propres à garantir la profitabilité des firmes. En agriculture, il s'agit de la libéralisation du secteur et de la privatisation des moyens de productions, dont la terre.

En nous basant sur l'analyse de Barzel (1997), nous distinguons trois types de droits de propriété qui peuvent être cédés lors de la privatisation d'un actif : le droit au bénéfice engendré par l'actif, le droit d'usage et le droit d'aliénation - les deux derniers étant aussi entendus comme droit de contrôle sur l'actif.

\section{Droits de propriété privés, incitations et efficience des marchés}

Les analyses théoriques menées en économie du développement ont largement abordé la question de l'intérêt économique de la privatisation de la terre. L'allocation de droits de propriété, en particulier s'ils sont individuels, permet tout d'abord de sécuriser la propriété foncière et, de ce fait, encourage l'investissement (Deiniger et Feder, op. cit.). En effet, l'incertitude sur les rendements 
de l'investissement, créée par la possibilité de hold-up ex post du produit de cet investissement par un tiers qui revendiquerait la propriété du bien, dissuade les producteurs à engager des frais qui ne pourraient pas être recouvrables en cas de litige.

La littérature sur la firme et sa restructuration pendant une période de transition vers une économie de marché propose un autre intérêt à la privatisation de l'ensemble des actifs des fermes d'État. En effet, elle démontre que les liens entre l'État (qui, pour le cas des fermes d'État, dispose du droit de contrôle et du droit au bénéfice) et l'unité de production impliquent l'adoption d'objectifs souvent différents de ceux garantissant une efficacité économique (Shleifer et Vishny, 1994), comme le maintien de l'emploi, par exemple : comme la privatisation alloue en particulier le droit de contrôle de la firme à des agents privés, les décisions de production et d'investissement sont plus efficaces. En revanche, en utilisant le même cadre théorique, certaines analyses mettent en évidence l'importance de la méthode de privatisation. En particulier, la cession de parts aux insiders, c'est-à-dire les employés de l'entreprise, au moment de la privatisation de celle-ci, a été largement critiquée : elle peut en effet favoriser le détournement d'actifs au profit des managers qui parviennent à récupérer le contrôle de la firme, et aggrave le problème de contrainte budgétaire molle, l'État refinançant alors des entreprises insolvables (Debande et Friebel, 1995).

Enfin, si les propriétaires privés disposent d'un droit d'aliénation, la privatisation permet une allocation efficace des moyens de production entre individus hétérogènes. En particulier pour ce qui concerne la propriété foncière (Deiniger et Feder, op. cit. ; Otsuka, 2007), la transférabilité de la terre, permanente ou transitoire, encourage la réallocation de la propriété, sous forme au moins de droit d'usage, aux unités relativement les plus productives. Sous l'hypothèse que les petites exploitations, à savoir les exploitations familiales (commerciales ou de subsistance), ont un avantage comparatif relativement aux grandes - du fait de coûts de supervision de la main-d'œuvre moins importants car elles n'ont pas d'employés (Allen et Lueck, 1998), même si cet avantage peut être contrebalancé en fonction des imperfections du marché du crédit (Feder, 1985) - les grandes entreprises d'État devraient réduire leur taille, et on devrait voir émerger, sur la base des anciennes exploitations ou créées ex nihilo, des exploitations individuelles de taille plus petite. À nouveau, le mode de privatisation choisi devrait influencer cette réallocation. En particulier, dans le cas de la privatisation des fermes collectives ou d'État par cession aux insiders, l'analyse théorique montre que, même inefficace, l'entreprise ne sera pas nécessairement rachetée par des outsiders (personnes extérieures à l'entreprise), généralement supposés plus efficaces dans leur gestion de l'entreprise, car la coalition créée par les insiders augmente leur prix de vente de réserve (Aghion et Blanchard, 1998). De même, certaines analyses de la réallocation des ressources dans les économies en transition (Bolton et Roland, 1992) soulignent que, dans le cas d'absence d'épargne préalable au niveau national, le transfert de propriété s'avère compliqué.

\section{Spécificités}

Pour le cas des pays en développement, Binswanger et al. (1995), entre autres, analysent l'émergence de droits de propriétés privés individuels comme résultant de l'augmentation de la rareté de la terre, et donc de la pression sur le foncier qui entraîne une augmentation de la valeur implicite de la terre (en l'absence de marchés), et donc un coût d'opportunité élevé à la sécurisation de la ressource foncière. L'individualisation des droits de propriété est ainsi décrite comme un phénomène endogène au processus de développement des économies. L'intervention des autorités publiques ou d'éléments extérieurs à ce processus 
engendrent des trajectoires non linéaires. De plus, la distribution initiale des droits de propriété sur la terre est avancée comme élément déterminant de l'évolution (Deininger, 2002).

Les pays en transition diffèrent partiellement de ce schéma en ce sens que l'on part d'un ensemble cohérent d'institutions basées sur la propriété collective ou d'État, suivi d'une rupture qui est la privatisation, puis d'une évolution vers de nouvelles structures de production par réallocation des facteurs. On ne peut dès lors pas faire abstraction des conditions initiales de la période de transition, et de leurs caractéristiques, dont certaines études ont souligné le caractère déterminant (Macours et Swinnen, op. cit., pour ce qui est du secteur agricole par exemple). En particulier, les économies en transition ont hérité d'une structure de production agricole spécifique et d'un secteur agricole souvent composé d'entreprises d'État et collectives. Dès lors, la réforme foncière comporte, au moins partiellement, une dimension organisationnelle due à la privatisation des structures de production. Contrairement à certains pays en développement où les réformes foncières sont autonomes, et peuvent consister, par exemple, en une simple opération de titrisation de la terre sur la base de l'existant, les réformes foncières dans les pays en transition ne peuvent pas être analysées en dehors de l'ensemble des réformes visant à la restructuration des exploitations agricoles héritées de la période socialiste : dès lors, la distinction entre les différents types de propriété de la terre et de l'outil de production, à savoir privé, collectif ou d'État, et la concentration de la propriété de ces capitaux, ont été déterminantes dans les trajectoires observées durant la transition et ont influencé les choix de réformes (Swinnen, 1999).

\section{Privatisations : des procédés multiples}

La réallocation des propriétés collectives et étatiques aux citoyens pouvait se faire selon trois modalités : la restitution, la redistribution ou une combinaison des deux. - La restitution consistait à restaurer la propriété aux propriétaires originels, c'està-dire ceux qui possédaient la terre avant la collectivisation forcée. En général, en raison du laps de temps important écoulé entre cette collectivisation et le début des réformes foncières, cette restitution devait se faire aux héritiers, souvent les petitsenfants des propriétaires initiaux.

- La redistribution consistait en une distribution de parts de terre ou de capital, sous la forme d'actions, aux citoyens. Elle pouvait se faire gratuitement ou aux enchères, à tout citoyen ou en premier lieu aux travailleurs de fermes collectives et étatiques.

La restitution a été choisie par tous les pays d'Europe centrale et orientale où la propriété de la terre était légalement encore privée en 1989. L'Albanie, tout comme les pays issus de l'Union soviétique, qui avaient nationalisé les terres, ont opté pour la redistribution. Pour ces derniers, de plus, bien que la restitution apparaisse comme le procédé de réallocation le plus juste, elle semblait irréaliste, puisqu'il s' agissait de retrouver qui étaient les propriétaires soixante-dix ans auparavant (Lerman, 2001).

Un autre argument contre la restitution était la volonté d'éviter des divisions ethniques dans les zones sensibles (par exemple dans la région Transniestra en Moldavie à la frontière avec l'Ukraine) et la restauration des terres aux étrangers qui les possédaient avant la collectivisation (par exemple les Allemands en Moldavie) (Gorton et White, 2003). Dans certains pays, la restitution a été remplacée, ou accompagnée par la compensation, c'est-à-dire l'attribution de bons de compensation à la place des parcelles initiales (Buckwell et Davidova, 1999). Deux pays optèrent pour une restitution accompagnée d'une redistribution, la Hongrie et la Roumanie. En particulier, les pays dans lesquels la 
concentration était forte avant la collectivisation ont, pour des raisons d'équité dans la distribution de la propriété, pu opter pour une redistribution au moins partielle (Swinnen, 1999) ${ }^{1}$.

La privatisation des fermes collectives et d'État pouvait donc impliquer une fragmentation de leurs moyens de production et un démantèlement total de ces entités. Le capital d'exploitation hors foncier était, dans la plupart des cas, transformé en actions dont la redistribution a fait l'objet de nombreux débats (Swinnen, 1999) : le principe général adopté a été celui de la cession de ces titres aux anciens employés des fermes. Parfois, comme en Bulgarie (Davidova et al., 1997), les gouvernements ont opté pour la « liquidation » de l'actif, c'està-dire sa redistribution physique. Cette politique était censée promouvoir la restructuration des unités de production privatisée en diminuant les coûts de sortie pour les récipiendaires de l'actif. La dimension organisationnelle des réformes agricoles, dont celle foncière, n'est donc pas à négliger.

\section{Après deux décennies Un bilan mitigé}

\section{Des marchés fonciers imparfaits et une propriété fragmentée}

L'activité des marchés fonciers pendant la période de transition a en général été faible. Par exemple, environ $0,2 \%$ seulement de la surface agricole utilisée de tout le pays était échangée par vente en République Tchèque entre 1993 et 2001 (Latruffe et Le Mouël, 2006). Plusieurs raisons expliquent cette faible activité.

- Tout d'abord, les échanges de terres étaient interdits sous le régime communiste : par

1. De plus amples détails sur les choix de réformes selon les pays sont disponibles dans Buckwell et Davidova (1999) pour les pays d'Europe centrale et orientale, et dans Lerman (2009) pour les pays de l'Union soviétique. conséquent, les réformes foncières impliquaient en premier lieu la création des institutions nécessaires à ces échanges et inexistantes jusque-là (cadastre, bureaux d'inscription au cadastre, bureau d'estimation de la valeur foncière, etc.). Cette création de toutes pièces n'a pas été rapide, et il a également fallu du temps aux propriétaires pour s'habituer à ce nouveau contexte institutionnel.

- Ensuite, dans le cas de la réallocation des terres par attribution d'actions, des droits de propriété virtuels ont été créés : les individus bénéficiaires ont tout d'abord dû comprendre le principe de ce mode de privatisation et apprendre comment transformer ces morceaux de papier en parcelles physiques (Ferenczi, 2005). Les procédures de conversion étaient elles-mêmes souvent complexes (Graefen, 2002). De plus, les propriétaires ont souvent été réticents à vendre leurs terres, pour des raisons psychologiques ou économiques ${ }^{2}$, mais également pour des raisons administratives (qui peuvent également s'apparenter à des raisons économiques si l'on considère les coûts de transaction qui en résultent). Ces raisons administratives créent des imperfections de fonctionnement du marché foncier. Après presque vingt ans de réformes foncières, la restitution des terres n'est toujours pas finie, pour plusieurs raisons : les héritiers de nombreux propriétaires décédés n'ont pas été identifiés ; les limites physiques de certaines parcelles n'ont pas encore pu être établies avec certitude ; les procédures d'inscription aux cadastres sont complexes et longues, ce qui rend ceux-ci encore incomplets. Les phénomènes de corruption sont courants, et les modifications des lois foncières sont fréquentes, ce qui rend les droits de propriété incertains.

- Enfin, les vendeurs et acheteurs subissent divers coûts lors des transactions (coûts d'identification des parcelles, d'estimation

2. Voir la section suivante «Une évolution inattendue vers une structure agricole duale ». 
de la valeur, d'inscription au cadastre, etc.), qui sont très élevés (Latruffe et Le Mouël, 2006). Par exemple, en Roumanie ces coûts étaient estimés à 10-30\% de la valeur de la transaction, et diverses enquêtes montrent que ces coûts de transaction sont une raison majeure de non-participation au marché foncier (Swinnen et Vranken, 2005). À côté des imperfections sur le marché foncier, des imperfections sur le marché du crédit empêchent les achats de terre. Les taux d'intérêt des emprunts sont élevés, en raison d'un contexte économique risqué, d'une absence de compétences des nouvelles banques et du manque d'expérience des agriculteurs en tant que demandeurs (le marché du crédit n'existait pas sous le régime communiste) $)^{3}$.

Dès lors, les analyses menées sur les marchés du faire-valoir indirect sont moins pessimistes : en effet, les transactions de location et métayage nécessitent des apports financiers moins conséquents, et sont réversibles. L'activité du marché du faire-valoir indirect semble pourtant tirée par les firmes agricoles ayant émergé dans la transition : ces dernières disposent de peu de terres en propriété, et sont pratiquement les seules à louer des parcelles. Pour le cas de la République Tchèque, Swinnen et Vranken (op. cit.) notent que ces exploitations utilisent $60 \%$ de la surface agricole du pays et louent $99 \%$ de ces terres. Pourtant, même si la location de terre par de petites exploitations ne représente qu'une faible part de l'activité, les auteurs conviennent que les chiffres peuvent être largement sous-estimés, du fait d'échanges informels, même si l'évolution semble aller vers une sécurisation de contrats.

Les réformes foncières n'ont donc pas entièrement réussi à créer un marché foncier qui fonctionne, les imperfections étant

3. En outre les banques acceptent rarement les terres agricoles comme garantie d'emprunt (Swinnen et Gow, 1999 ; Latruffe, 2005). encore très nombreuses. De plus, elles ont créé une propriété très fragmentée. Cette fragmentation trouve son origine dans les réformes elles-mêmes, puisqu'elles ont redistribué ou restitué des micro-parcelles : en particulier, en cas de structure fragmentée de la propriété avant la Seconde Guerre mondiale, le processus de restitution a contribué à faire émerger la fragmentation (Deininger, 2002). Puis les marchés fonciers sous-développés n'ont pas permis un regroupement de la propriété. Par exemple, une enquête de 2005 indique que les terres en fermage dans les grandes exploitations (entreprises privées ou coopératives) slovaques étaient en moyenne possédées par 789 propriétaires individuels (Latruffe et Davidova, op. cit.). En Bulgarie, une enquête de 2003 montre que les ménages agricoles possédaient en moyenne six parcelles, avec une taille moyenne de 0,6 hectare par parcelle (Vranken et al., 2004). En République Tchèque il $\mathrm{y}$ aurait actuellement 3,5 millions de très petits propriétaires (Latruffe et al., 2008). Certains pays ont mis en place un remembrement ${ }^{4}$, mais celuici est rendu très difficile par les identifications peu précises des limites des parcelles et par les inscriptions aux cadastres encore manquantes. Par exemple, en République Tchèque, seulement $8 \%$ des terres étaient remembrés au milieu des années 2000 (Latruffe et al., op. cit.).

\section{Une évolution inattendue vers une structure agricole duale}

Les réformes foncières devaient permettre la création d'exploitations de taille moyenne, comme celles prévalant dans les pays occidentaux. En effet, comme expliqué dans la première partie «Nécessité et modalités des réformes foncières », la création de droits de propriété et le démantèlement des fermes collectives et étatiques devaient faci-

4. En particulier la République Tchèque, la Bulgarie, la Hongrie et la Roumanie (Swinnen et Vranken, 2005) 
liter l'échange de parcelles, et permettre aux exploitations les moins efficaces de quitter le secteur agricole et aux exploitations les plus efficaces de se créer ou de s'agrandir. D'un point de vue économique, il était attendu que les petites exploitations, en dessous de la taille critique nécessaire aux économies d'échelle, et les très grandes exploitations, souffrant de coûts de transaction élevés et de déséconomies d'échelle, disparaissent au profit d'exploitations individuelles de taille moyenne comme dans les pays occidentaux (Kydd et al., 1997). Celles-ci auraient été créées de toutes pièces (les exploitations de novo) ou auraient résultées d'un agrandissement d'exploitations existantes, et auraient ainsi cumulé les deux avantages d'économies d'échelle et de faibles coûts de transaction. Bien que la diversité des structures agricoles selon les pays considérés ne doit pas être négligée (Swinnen, 2010), nous pouvons tirer quelques conclusions de l'analyse menée.

Presque vingt ans après le début des réformes, le développement d'exploitations de taille moyenne n'est pas pour autant aussi nettement observable que prévu.

\section{- Tout d'abord, le démantèlement des fermes} collectives ou d'État n'a pas été systématique. Si, dans les textes, ces deux statuts juridiques ont disparu, dans les faits les exploitations de très grande taille avec un caractère collectif existent toujours, même si elles sont moins nombreuses. Les fermes d'État ont souvent été rachetées par quelques-unes de leurs managers $^{5}$, qui les ont transformées en entreprises privées (compagnies à responsabilité limitée, sociétés par actions). Ces entreprises utilisent des centaines d'hectares, et emploient des centaines de salariés. Certaines, acquises par des fonds privés initialement investis dans des activités non agricoles,

5. En particulier dans les pays issus de l'Union Soviétique (Mathijs et Swinnen, 1998). prospèrent (Rylko et al., 2008). Quant aux fermes collectives, elles ont en général gardé leur caractère collectif ${ }^{6}$, en prenant le statut juridique de coopératives : possédées par de nombreux associés, gérées par un conseil élu, souvent selon le principe de « un homme/une voix », elles utilisent des milliers d'hectares et emploient des milliers de salariés. Les raisons de ce maintien des grandes exploitations sont multiples (Rizov et al., 2001 ; Slangen et al., 2004 ; Latruffe et Davidova, op. cit.). Lors de la privatisation, les individus ayant reçu des parts de capital et de terre (des actions) pouvaient choisir :

i) de partir avec ces parts,

ii) de les revendre contre argent aux exploitations qui succéderaient aux fermes collectives et d'État,

iii) ou de les laisser dans ces fermes en tant que propriétaires, devenant ainsi actionnaires ou loueurs.

Choisir entre la première et la troisième option revenait à décider entre créer sa propre exploitation individuelle (les exploitations de novo) avec le capital et la terre reçus, ou rester membre d'une exploitation de type collectif. Plusieurs auteurs ont proposé des cadres conceptuels d'analyse pour expliquer le choix des individus bénéficiaires d'actifs, comme par exemple la comparaison des utilités des deux options, nettes des coûts de sortie (coûts pour récupérer ses parts et coûts de création d'une nouvelle exploitation). Du point de vue empirique, ils ont mis en avant divers facteurs décisionnels : caractéristiques des individus, profitabilité de la ferme collective ou étatique, risque de prix et de production, coûts de transaction sur le marché foncier, capital social (Mathijs et Swinnen, 1998 ; Rizov et al., op. cit. ; Slangen et al., op. cit.). De plus, ils insistent sur les éléments institutionnels déter-

6. Sauf en Albanie, Arménie et Lettonie (Mathijs et Swinnen, 1998). 
minant le choix des agents : ils montrent que la décollectivisation et l'émergence d'exploitations individuelles privées sont observées quand la distribution de la terre aux ouvriers a été privilégiée plutôt que la restitution, et quand les coûts de sortie sont faibles, notamment en fonction de la régulation et l'efficacité de la mise en œuvre des réformes (Mathijs et Swinnen, op. cit.). Le poids des exploitations individuelles dans le contexte d'avant réforme, interprété comme un indicateur du développement de l'entreprenariat, est aussi explicatif de leur progression durant la transition (Rizov et al., op. cit.).

- Ensuite, l'environnement général dans lequel les agents évoluent est décisif : niveau de la corruption, protection des droits ou climat de confiance (Slangen et al., 2004). Ce sont ensuite les héritiers, en général les petits-enfants, des anciens propriétaires qui ont reçu les droits de propriété des parcelles restituées par les réformes foncières. Or ils vivent, pour la plupart, à la ville et n'ont aucun lien avec l'agriculture. Créer d'euxmêmes une exploitation individuelle aurait été trop coûteux ; trouver un acheteur aurait également supposé de forts coûts de transaction, en raison des imperfections sur le marché foncier. L'option la moins coûteuse pour ces héritiers était donc de laisser leur parcelle en location à la nouvelle exploitation (entreprise ou coopérative). Amblard et Colin (2006) rapportent par exemple qu'entre 20 et $50 \%$ des propriétaires d'exploitations de type collectif (les sociétés agricoles) en Roumanie sont des propriétaires urbains. Les anciens employés des fermes collectives et étatiques, ayant reçu une part de ces exploitations lors des redistributions, faisaient face aux mêmes options. La différence était que ces employés, ayant déjà des compétences agricoles, auraient pu démarrer une exploitation individuelle plus facilement. Néanmoins, leur part reçue était en général trop petite, ce qui aurait nécessité l'achat de parcelles supplémen- taires, une transaction coûteuse sur le marché foncier imparfait. De plus, pour beaucoup d'entre eux, faire partie d'une coopérative pouvait leur apporter des avantages supplémentaires (des services comme la restauration collective, ou des avantages en nature), et était souvent l'option qui convenait le plus à leurs convictions personnelles, héritées de la période socialiste (agriculture collective). On peut également souligner une autre raison majeure allant à l'encontre de la création d'exploitations individuelles de novo : le risque. Créer sa propre exploitation pendant la première décennie de transition était plutôt risqué : la situation économique était mauvaise, les prix et les politiques instables, et les infrastructures d'amont et d'aval (qui n'existaient pas sous le régime communiste) lentes à se créer.

- Enfin, la dernière raison que l'on peut proposer pour expliquer le faible retrait des terres individuelles des fermes collectives et étatiques, concerne les difficultés, et donc le coût, de ce retrait : après des décennies de collectivisation et d'absence de cadastre, les limites des parcelles étaient difficiles, voire impossibles, à retrouver ; de plus, certaines parcelles se trouvaient au milieu des terres exploitées par les fermes collectives ou d'État, qui devaient donc trouver une parcelle de substitution identique à un autre endroit ; enfin, après deux générations, les héritiers d'une seule parcelle étaient souvent nombreux, ce qui rendaient les décisions de vente difficiles.

À côté du maintien des exploitations de très grande taille, les exploitations de très petite taille, généralement des exploitations de semi-subsistance très peu tournées vers la commercialisation de leur production (les « microfundia » de Pouliquen, 1999), se sont fortement maintenues, voire développées pour les cas de l'Albanie et la Roumanie (Swinnen, 2010).

La raison principale est la situation économique pendant la transition, notamment un 
taux de chômage élevé et une inflation forte. Dans ces conditions, il n'y avait pas d'alternative à l'agriculture dans les zones rurales, et la terre elle-même était considérée comme un filet de sécurité, pour les ruraux mais également pour les ouvriers ayant perdu leur emploi lors de l'effondrement du secteur industriel étatique (Bafoil et al., 2003).

Une deuxième raison pouvant être évoquée concerne surtout la Pologne : dans ce pays, la retraite agricole (du fonds de pension KRUS) était beaucoup plus généreuse que la retraite des ouvriers des autres secteurs, et les conditions d'obtention étaient peu contraignantes (il suffisait de posséder un hectare de terre pendant plusieurs années de cotisation). Cette politique a incité de nombreux agriculteurs à s'accrocher à leurs petites parcelles de terre.

Enfin, le dernier aspect pouvant expliquer le maintien des petites exploitations a trait à la psychologie : après des décennies passées sous un régime qui n'autorisait pas la propriété individuelle, les terres possédées avaient non seulement une valeur économique, mais également une valeur psychologique forte. Les vendre ou les louer à d'autres personnes rappelait trop la période de collectivisation forcée. Pour autant, l'agriculture de semi-subsistance menée sur des exploitations de petite taille a été aussi entendue comme une stratégie de survie choisie et nécessaire, et qui ne serait de plus pas affectée par des opérations de consolidation de la propriété foncière (Davidova $e t$ al., 2009).

Les réformes foncières dans les pays d'Europe centrale et orientale n'ont pas eu l'effet escompté en termes de structures agricoles. À la place de l'émergence attendue de structures de taille moyenne, la transition a donné lieu à un secteur agricole dual dans la plupart des pays (Rozelle et Swinnen, 2004), où de très nombreuses exploitations de petite taille coexistent avec des exploitations de taille moyenne, ainsi qu'avec de très grandes unités, peu nombreuses mais utilisant la majeure partie des terres agricoles comme le montrent les tableaux 2 et 3. Même s'il est difficile de comparer, en raison de sources et de modalités de calcul différentes, avec à la situation de début de transition présentée dans le tableau 1 , on peut noter que le développement en un secteur agricole dual est particulièrement marqué en République Tchèque.

Tableau 1. Répartition des exploitations selon leur statut juridique et leur taille au début de la transition

\begin{tabular}{|c|c|c|c|c|}
\hline & \multirow{2}{*}{$\begin{array}{l}\text { Part de la surface } \\
\text { exploitée par } \\
\text { les exploitations } \\
\text { individuelles } \\
\text { en } 1990(\%) \text { * }\end{array}$} & \multicolumn{3}{|c|}{$\begin{array}{l}\text { Répartition des exploitations selon leur taille : part } \\
\text { dans le nombre d'exploitations du pays en 1994/1995 (\%)** }\end{array}$} \\
\hline & & $\begin{array}{l}\text { Exploitations } \\
\text { de moins } \\
\text { de } 5 \text { hectares }\end{array}$ & $\begin{array}{l}\text { Exploitations entre } \\
5 \text { et } 100 \text { hectares } \\
\text { (exclus) }\end{array}$ & $\begin{array}{c}\text { Exploitations } \\
\text { de } 100 \text { hectares } \\
\text { ou plus }\end{array}$ \\
\hline Bulgarie & 13 & 30 & 6 & 64 \\
\hline République Tchèque & 5 & 1 & 5 & 92 \\
\hline Estonie & 6 & & & \\
\hline Lettonie & 5 & & & \\
\hline Lituanie & 9 & & & \\
\hline Hongrie & 6 & 22 & 20 & 58 \\
\hline Pologne & 77 & & & \\
\hline Roumanie & 12 & 45 & 10 & 45 \\
\hline Slovénie & 92 & & & \\
\hline Slovaquie & 5 & 2 & 2 & 96 \\
\hline
\end{tabular}


Tableau 2. Répartition des exploitations selon leur taille en 2007

\begin{tabular}{l|c|c|c|c|c|c}
\hline & \multicolumn{2}{|c|}{ Part dans le nombre d'exploitations du pays (\%) } & \multicolumn{3}{|c}{ Part de la surface du pays exploitée (\%) } \\
\cline { 2 - 7 } & $\begin{array}{c}\text { Exploitations } \\
\text { de moins } \\
\text { de 5 hectares }\end{array}$ & $\begin{array}{c}\text { Exploitations } \\
\text { entre 5 } \\
\text { et 50 hectares } \\
\text { (exclus) }\end{array}$ & $\begin{array}{c}\text { Exploitations } \\
\text { de 50 hectares } \\
\text { ou plus }\end{array}$ & $\begin{array}{c}\text { Exploitations } \\
\text { de moins } \\
\text { de 5 hectares }\end{array}$ & $\begin{array}{c}\text { Exploitations } \\
\text { entre 5 } \\
\text { et 50 hectares } \\
\text { (exclus) }\end{array}$ & $\begin{array}{c}\text { Exploitations } \\
\text { de 50 hectares } \\
\text { ou plus }\end{array}$ \\
\hline Bulgarie & 95 & 4 & 1 & 13 & 8 & 78 \\
Rép. tchèque & 50 & 33 & 17 & 1 & 5 & 93 \\
Estonie & 36 & 53 & 11 & 6 & 29 & 64 \\
Lettonie & 41 & 54 & 5 & 10 & 50 & 40 \\
Lituanie & 61 & 36 & 3 & 16 & 41 & 43 \\
Hongrie & 89 & 9 & 2 & 1 & 14 & 85 \\
Pologne & 68 & 31 & 1 & 10 & 15 & 76 \\
Roumanie & 90 & 10 & 0,4 & 20 & 56 & 23 \\
Slovénie & 59 & 40 & 1 & 35 & 24 & 41 \\
Slovaquie & 87 & 9 & 4 & 27 & 67 & 6 \\
\hline
\end{tabular}

Source : Eurostat, Structures des exploitations agricoles, 2011. http://epp.eurostat.ec.europa.eu/portal/page/portal/statistics/search_database

Tableau 3. Répartition des exploitations selon leur statut juridique en 2007

\begin{tabular}{l|c|c|c|c}
\hline & \multicolumn{3}{|c|}{ Part dans le nombre d'exploitations du pays (\%) } & \multicolumn{2}{|c}{ Part de la surface du pays exploitée (\%) } \\
\hline & $\begin{array}{c}\text { Exploitations } \\
\text { individuelles }\end{array}$ & $\begin{array}{c}\text { Exploitations de } \\
\text { personne morale } \\
\text { ou en groupement }\end{array}$ & $\begin{array}{c}\text { Exploitations } \\
\text { individuelles }\end{array}$ & $\begin{array}{c}\text { Exploitations de } \\
\text { personne morale } \\
\text { ou en groupement }\end{array}$ \\
\hline Bulgarie & 99 & 1 & 47 & 53 \\
Rép. tchèque & 93 & 7 & 29 & 71 \\
Estonie & 94 & 6 & 52 & 48 \\
Lettonie & 99 & 1 & 91 & 9 \\
Lituanie & 100 & 0,2 & 86 & 14 \\
Hongrie & 99 & 1 & 48 & 52 \\
Pologne & 100 & 0,2 & 90 & 10 \\
Roumanie & 100 & 0,5 & 65 & 35 \\
Slovénie & 100 & 0,2 & 95 & 5 \\
Slovaquie & 97 & 3 & 20 & 80 \\
\hline
\end{tabular}

Source : Eurostat, Structures des exploitations agricoles, 2011. http://epp.eurostat.ec.europa.eu/portal/page/portal/statistics/search_database

\section{Perspectives}

Pour les pays en développement, la littérature considère que l'émergence de droits de propriété privés individuels sur la terre est la conséquence de l'augmentation de la pression foncière. Pour les pays d'Europe centrale et orientale, l'héritage d'un système productif basé sur la propriété collective et celle d'État accroît l'importance des conditions initiales de la période de transition. Dès lors, les réformes foncières ont été l'un des éléments d'un ensemble de politiques, en particulier de la politique industrielle, visant à restructurer l'appareil productif hérité de la période socialiste.

Même si les gouvernements des pays d'Europe centrale et orientale avaient conscience de la probable complexité des réformes foncières à venir, ils n'avaient sans doute pas anticipé une telle lenteur, ni la dimension psychologique qui conditionnait ces transactions. Cette lenteur a eu une conséquence inattendue sur l'évolution des structures agricoles, avec la création d'un régime structurel dual. Aujourd'hui, presque vingt ans après le début des 
réformes foncières, les marchés fonciers sont toujours imparfaits, ce qui consolide cette bi-polarité.

Néanmoins, la persistance des petites exploitations de semi-subsistance et des grandes structures employant des centaines d'employés, parfois inutilement, a sans doute permis d'éviter un désastre social pendant la difficile période de transition économique, en garantissant un niveau de vie minimum à des millions de personnes dans les zones rurales. Pouliquen (1999) estime que cela a amorti le coût social de la transition. Ayant bien conscience de cet enjeu social, la Commission européenne a fini par faire des concessions sur ce point lors des négociations d'adhésion, en permettant aux États entrants d'apporter à leurs agriculteurs des aides complémentaires aux aides de la Politique agricole commune (PAC). La question de l'agriculture était une question cruciale pendant la phase de négociations précédant l'élargissement de l'Union européenne aux PECO. En effet, non seulement l'agriculture était un secteur primordial dans ces pays, elle pesait également lourd dans le budget européen (45\%) en raison des subventions aux agriculteurs. L'adhésion signifiait donc, pour ces pays, recevoir une part du gâteau constitué par ces subventions agricoles généreuses, alors que pour la Commission et pour les anciens États membres, l'élargissement impliquait une augmentation de la part budgétaire de l'agriculture, et donc une plus grande contribution de certains pays (comme la France et l'Allemagne) au budget européen. De plus, il pouvait paraître risqué de permettre aux agriculteurs des nouveaux États membres de recevoir les mêmes aides de la PAC que ceux des anciens, car cela pouvait freiner le processus de restructuration et consolider les structures inefficaces. La décision finale a donc été d'augmenter graduellement le montant des aides PAC accordées aux agriculteurs des nouveaux États membres, jusqu'à 2013 où le montant sera le même que celui reçu par les agriculteurs des anciens États membres. Néanmoins, face à l'enjeu social que constituait le maintien du secteur agricole, les gouvernements des nouveaux États membres ont été autorisés à verser des subventions complémentaires (top-ups) aux aides de la PAC, et des aides spécifiques aux petites exploitations de semi-subsistance.

Une réallocation de masse des facteurs de production, intra-sectorielle et inter-sectorielle, était attendue dans les pays d'Europe centrale et orientale. Or, elle n'a pas été massive, et l'apport de subventions de la PAC peut freiner encore plus cette réallocation, puisqu'en présence de subventions qui retardent la restructuration, c'est la notion de vitesse de transition qui est en jeu. Néanmoins, Ciaian et Swinnen (2006) ainsi que Bazin (2007) estiment que les subventions vont justement permettre aux grandes et moyennes unités de se moderniser et d'améliorer leur compétitivité afin d'évoluer selon le modèle occidental, alors que les petites exploitations de subsistance seraient plutôt désavantagées par le système de subventions, qui pourrait compromettre leur survie. 


\section{RÉFÉRENCES BIBLIOGRAPHIQUES}

Aghion P., Blanchard O. (1998). On privatization methods in Eastern Europe and their implications. Economics of Transition, Vol. 6, $\mathrm{n}^{\circ}$ 1, p. 87-99.

Allen D.-W., Lueck D. (1998). The nature of the farm. Journal of Law and Economics, Vol. 41, p. 343-386.

Amblard L., Colin J.-P. (2006). Dimension organisationnelle et pratiques contractuelles foncières : les sociétés agricoles en Roumanie. Économie rurale, Vol. 293, p. 55-71.

Bafoil F., Guyet R., Thardy V., Lharidon L. (2003). Qui est paysan en Pologne en 2002 ? Le Courrier des Pays de l'Est, Vol. avril, p. 28-45.

Barzel Y. (1997). Economic Analysis of Property Rights. Cambridge, Cambridge University Press. $2^{\mathrm{e}}$ édition.

Bazin G. (2007). Politique agricole commune à l'Est - Premiers résultats. Le Courrier des Pays de l'Est, Vol. 1063, p. 11-21.

Binswanger H., Deininger K., Feder G. (1995). Power, distortions, revolt and reform in agricultural land relations. In Behrman J., Srinivasan T.-N. (Eds.), "Handbook of Development Economics", Elsevier Science, Vol. 3B, p. 2659-2772.

Binswanger H., Rosenzweig M. (1986). Behavioral and material production conditions in agriculture. Journal of Development Studies, Vol. 22, n 3, p. 503-539.

Bolton P., Roland G. (1992). Privatization in Central and Eastern Europe. Economic Policy, Vol. 7, n 15, p. 276-309.

Brada J., King A. (1993). Is private farming more efficient than socialized agriculture. Economica, Vol. 60, ${ }^{\circ}$ 237, p. 41-56.

Buckwell A., Davidova S. (1999). Land reform and de-collectivisation in CEECs: Some conceptual issues. Bulgarian Journal of Agricultural Science, Vol. 5, p. 325-336.
Chauveau J.-P., Lavigne Delville P. (2002). Quelles politiques foncières intermédiaires en Afrique de l'Ouest francophone ? In Lévy M. (éd.), « Comment réduire pauvreté et inégalités en Afrique. Pour une méthodologie des politiques publiques », IRD-Karthala, Paris, France, p. 211-239.

Ciaian P., Swinnen J. (2006). Land market imperfections and agricultural policy impacts in the New UE Member states: A partial equilibrium analysis. American Journal of Agricultural Economics, Vol. 88, n 4, p. 799-815.

Civici A., Jouve A.-M. (2009). Enchâssement social et politique de la propriété foncière dans les Balkans. Options Méditerranéennes, série A, Vol. 82, p. 5-20.

Davidova S., Buckwell A. (1999). Land reform and de-collectivization in CEECs: some conceptual issues. Bulgarian Journal of Agricultural Sciences, Vol. 5, p. 325-336.

Davidova S., Buckwell A., Kopeva D. (1997). Bulgaria: Economics and politics of post-reform farm structures. In Swinnen J., Buckwell A., Mathijs E. (Eds.), "Agricultural privatisation, land reform and farm restructuring in Central and Eastern Europe”, Ashgate, Brookfield, États-Unis, p. 23-62.

Davidova S., Fredriksson L., Bailey A. (2009). Subsistence and semi-subsistence farming in selected EU new member states. Agricultural Economics, Vol. 40, $\mathrm{n}^{\circ} \mathrm{S} 1, \mathrm{p} .733-744$.

Debande O., Friebel G. (1995). Privatization, employment and managerial decision-taking. Mimeo, ECAREs, ULB.

Deininger K. (2002). Agrarian reforms in Eastern European Countries: Lessons from international experience. Journal of International Development, Vol. 14, p. 987-1003. 
Deininger K., Feder G. (2001). Land institutions and land markets. In Gardner B.-L., Rausser G.-C. (Eds.), "Handbook of Agricultural Economics”, Elsevier Science, Vol. 1A, p. 287-331.

Feder G. (1985). The relation between farm size and farm productivity: the role of family labor, supervision and credit constraints. Journal of Development Economics, Vol. 18, p. 297-313.

Ferenczi T. (2005). Promoting Strong Land Markets in Transition Economies. Department of Agricultural and Applied Economies, University of Wisconsin, ÉtatsUnis, Basis Brief $n^{\circ} 42$.

Gorton M., White J. (2003). Responses to the Decollectivisation Question in PostSoviet States: A Comparison of Ukraine, Moldova and the Baltic States. University of Plymouth Business School, RoyaumeUni, Discussion Paper ISBN 1- 84102111-3.

Graefen C. (2002). Land Reform and Land Fragmentation and Consequence for Rural Development in the CEE/CIS Countries. Présentation au Symposium International "Land fragmentation and land consolidation in Central and Eastern European Countries - A gate towards sustainable rural development in the New Millennium”, organisé par FAO, GTZ, FIG, ARGE Landentwicklung et TUM, Munich, Allemagne, 25-28 février.

Kydd J., Buckwell A., Morrison J. (1997). The role of the agriculture sector in the transition to a market economy in Central and Eastern Europe: An analytical framework. In Kydd J., Davidova S., Mackay M. Mech T. (Eds.), "The Role of Agriculture in the transition Process towards a Market Economy”, Economic Studies $\mathrm{n}^{\circ}$ 9, FAO et Nations-Unies, Suisse, p. 1-20.

Kornai J. (1980). Economics of Shortage. Amsterdam, Pays-Bas, North Holland Publishing Co.

Latruffe L. (2005). The impact of credit market imperfections on farm investment in Poland. Post-Communist Economies, Vol. 17, n 3, p. 349-362.

Latruffe L., Davidova S. (2007). Corporate Farms in the New Member States: Is their Future Undermined by the CAP Single Area Payment? Projet du $6^{\mathrm{e}}$ PCRD IDEMA ("Impact of decoupling and modulation in the enlarged Union: a sectoral and farm level assessment"), Deliverable 22.

Latruffe L., Doucha T., Le Mouël C., Medonos T., Voltr V. (2008). Capitalisation of the government support in agricultural land prices in the Czech Republic. Agricultural Economics - Czech, Vol. $54, \mathrm{n}^{\circ} 10$, p. 451-460.

Latruffe L., Le Mouël C. (2006). Description of Agricultural Land Market Functioning in Partner Countries. Projet du $6^{\circ}$ PCRD IDEMA ("Impact of decoupling and modulation in the enlarged Union: a sectoral and farm level assessment"), Deliverable 9.

Lerman Z. (2001). Agriculture in transition economies: from common heritage to divergence. Agricultural Economics, Vol. 26, n 2, p. 95-114.

Lerman Z. (2009). Land reform, farm structure, and agricultural performance in CIS countries. China Economic Review, Vol. 20, p. 316-326.

Macours K., Swinnen J. (2002). Patterns of agrarian transition. Economic Development and Cultural Change, Vol. 50, $\mathrm{n}^{\circ} 2$, p. 365-395.

Mathijs E., Swinnen J. (1998). The economics of agricultural decollectivisation in East Central Europe and the Former Soviet Union. Economic Development and Cultural Change, Vol. 47, $\mathrm{n}^{\circ} 1$, p. 1-26.

Maurel M. (2006). Héritages, réformes, institutions : un bilan de quinze années de transition. Revue d'Études Comparatives Est-Ouest, Vol. 37, n 1, p. 97-125.

Otsuka K. (2007). Efficiency and equity effects of land markets. In Gardner B.-L., Rausser G.-C. (Eds.), "Handbook of 
Agricultural Economics”, Elsevier Science, Vol. 3, p. 2671-2703.

Pollak R. (1985). A transaction cost approach to families and households. Journal of Economic Literature, Vol. 23, p. 581-608.

Pouliquen A. (1999). Élargissement agricole de l'Union européenne : le suremploi contre les excédents. Revue d'Études Comparatives Est-Ouest, Vol. 31, ${ }^{\circ} 1$, p. 233-259.

Rozelle S., Swinnen J. (2004). Success and failure of reform: insights from the transition of agriculture. Journal of Economic Literature, Vol. 42, $\mathrm{n}^{\circ}$ 2, p. 404-456.

Rizov M., Gavrilescu D., Gow H., Mathijs E., Swinnen J. (2001). Transition and enterprise restructuring: the development of individual farming in Romania. World Development, Vol. 29, p. $1257-1274$.

Rylko D., Khramova I., Uzun V., Jolly R. (2008). Agroholdings: Russia new agricultural operators. In Lerman Z. (Ed.), "Russia's Agriculture in Transition, Factor Markets and Constraints on Growth”, Lexington Books, p. 95-136.

Schmitt G. (1991). Why is the agriculture of advanced western countries still organised by family farms? Will this continue to be so in the future? European Review of Agricultural Economics, Vol. 18, p. 443-458.

Shleifer A., Vishny R.-W. (1994). Politicians and firms. Quarterly Journal of Economics, Vol. 109, p. 995-1025.
Slangen L., van Kooten C., Suchanek P. (2004). Institutions, social capital and agricultural change in central and eastern Europe. Journal of Rural Studies, Vol. 20, $\mathrm{n}^{\circ} 2$, p. $245-256$.

Swinnen J. (1999). The political economy of land reform choices in Central and Eastern Europe. Economics of Transition, Vol. 7, $\mathrm{n}^{\circ} 3$, p. 637-664.

Swinnen J. (2010). Reforms, globalization, and endogenous agricultural structures. Agricultural Economics, Vol. 40, $\mathrm{n}^{\circ} \mathrm{S} 1$, p. 719-732.

Swinnen J., Gow H. (1999). Agricultural credit problems and policies during the transition to a market economy in Central and Eastern Europe. Food Policy, Vol. 24, $\mathrm{n}^{\circ} 1$, p. 21-47.

Swinnen J., Vranken L. (2005). The Development of Rural Land Markets in Transition Countries. Rapport pour le Bureau Régional de Budapest, Hongrie, de la FAO pour l'Europe centrale et orientale, et la Faculté de Management de l'Université Agricole Slovaque à Nitra, Slovaquie.

Swinnen J., Vranken L. (2007). Patterns of Land Market Developments in Transition. LICOS Discussion Paper $\mathrm{n}^{\circ} 179$, Katholieke Universiteit Leuven, 42 p.

Vranken L., Noev N., Swinnen J. (2004). Fragmentation, abandonment, and coownership: transition problems of the Bulgarian land market. Quarterly Journal of International Agriculture, Vol. 43, $\mathrm{n}^{\circ} 4$, p. 391-408. 